\title{
Unterrichtsqualität zwischen Generik und Fachspezifik: Einführung in den Thementeil
}

\author{
Anna-Katharina Praetorius $(\mathbb{D} \cdot$ Andreas Nehring
}

Online publiziert: 13. Juli 2020

(C) Die Herausgeber und Springer Fachmedien Wiesbaden GmbH, ein Teil von Springer Nature 2020

Das Thema Unterrichtsqualität erfreut sich seit einigen Jahren in der allgemeinen wie auch in der fachspezifischen empirischen Bildungsforschung großer Beliebtheit. Verortet ist die Forschung zu Unterrichtsqualität in der allgemeinen Unterrichtsforschung sowie zum Teil auch in der fachspezifischen Unterrichtsforschung im Educational Effectiveness Paradigma, fokussiert also auf diejenigen Aspekte von Unterricht, die das Verfolgen extern gesetzter Erziehungs- und Bildungsziele unterstützen - in bisherigen empirischen Studien mit Schwerpunkt auf leistungsbezogenen Zielen. In anderen Teilen der fachspezifischen Unterrichtsforschung finden sich hingegen vorrangig theoretisch-konzeptuelle Arbeiten zum Thema.

Fachdidaktische Forschung zu Unterrichtsqualität nimmt in der Regel solche Aspekte von Unterrichtsqualität in den Blick, die für ein bestimmtes Fach Relevanz aufweisen (z.B. Brunner 2018; Maurer 2018). In der generisch ausgerichteten Unterrichtsqualitätsforschung hingegen liegt der Schwerpunkt auf der empirischen Untersuchung von Aspekten, die als über Fächer hinweg bedeutsam für die Qualität von Unterricht angenommen werden (Helmke 2015; Klieme 2019). Kritisiert wird dabei, dass der für empirische Studien notwendige Bezug zu konkreten Fächern bzw. Fachdidaktiken in der allgemeinen Unterrichtsforschung vorrangig für Mathematik sowie die naturwissenschaftlichen Fächer umgesetzt wurde (Kunter und Ewald 2016). Die wenigen existierenden Studien über Fächer hinweg zeigen, dass sowohl die untersuchten Unterrichtsaspekte als auch die empirischen Befunde nur

A.-K. Praetorius $(\bowtie)$

Institut für Erziehungswissenschaft, Universität Zürich, Freiestr. 36, 8032 Zürich, Schweiz

E-Mail: anna.praetorius@ife.uzh.ch

A. Nehring

Institut für Didaktik der Naturwissenschaften, Leibniz Universität Hannover, Am Kleinen

Felde 30, 30167 Hannover, Deutschland 
teilweise über Fächer hinweg generalisiert werden können (z. B. Blömeke und Olsen 2019; Praetorius et al. 2016).

Möchte man sich Unterrichtsqualität in einer fächervergleichenden Weise nähern, stellen sich viele Herausforderungen. So ist bislang beispielsweise kaum geklärt, wie und worin sich Fachspezifität konstituiert und inwiefern diese bei der theoretischen Fassung von Unterrichtsqualität eine Rolle spielt (siehe auch Praetorius und Charalambous 2018). Im Fächervergleich wird zudem deutlich, dass Unterrichtsqualität nicht nur von den gesetzten Zielen, sondern auch von den thematisierten Inhalten und den eingesetzten Methoden abhängt und entsprechend alleine aus diesem Grund Unterschiede zwischen Fachdidaktiken in der Auseinandersetzung mit Unterrichtsqualität zu erwarten sind. Diese Herausforderungen anzugehen ist aufwändig und anspruchsvoll. Dies gilt umso mehr, da auch die betreffenden Diskurse der verschiedenen Disziplinen und Fachdidaktiken bislang vielfach eher unverbunden nebeneinander stehen und daher eine für interdisziplinäre Kommunikation notwendige gemeinsame Sprache und Strukturierung des Gegenstands Unterricht nur bedingt gegeben ist. Das Potenzial, das sich aus einer stärker interdisziplinären Verknüpfung ergeben könnte, bleibt dadurch bislang weitgehend ungenutzt.

\section{Interdisziplinäre Arbeitsgruppe zur Fachspezifität von Unterrichtsqualität}

Um einen entsprechenden Dialog zu initiieren, wurde 2018 eine Arbeitsgruppe mit den am vorliegenden Thementeil beteiligten Personen ins Leben gerufen. Mit der Wahl der Fächer aus den Bereichen Naturwissenschaften (mit besonderem Fokus auf Chemie), Gesellschaftswissenschaften (Geschichte) und dem musisch-ästhetischen Bereich (Sport) wurde dabei gezielt ein kontrastierender Ansatz gewählt. Die Gruppe einigte sich initial auf eine gemeinsame Verständnisgrundlage für die Diskussionen zu Unterrichtsqualität über Fächer hinweg. Aufgrund der aktuellen Integration von generischen und fachspezifischen Aspekten von Unterrichtsqualität wurde hierzu das internationale Syntheseframework von Praetorius und Charalambous (2018) gewählt, das eine aktuelle Synthese bestehender Frameworks zu Unterrichtsqualität darstellt. Auftakt der gemeinsamen Arbeit stellte ein zweitägiger Workshop in Hannover dar. Vorrangige Ziele des Workshops waren (a) sich wechselseitig über die jeweiligen Ansätze zur Erforschung von Unterrichtsqualität in der eigenen Disziplin zu informieren, (b) zentrale Ergebnisse vorzustellen und (c) diese Ergebnisse in eine erste Relation zum Syntheseframework von Praetorius und Charalambous (2018) zu setzen. Zudem sollte im Workshop ein gemeinsames Vorgehen entwickelt werden, um den jeweiligen Forschungsstand systematisch aufzuarbeiten und in einem letzten Schritt über diesen Stand eine gemeinsame Synthese zu ermöglichen.

Im Rahmen der Diskussionen während des Workshops wurde rasch deutlich, dass sich die verwendeten Begrifflichkeiten, Strukturierungen und Schwerpunktsetzungen zwischen den Fächern deutlich unterschieden und diese jeweils eine unterschiedliche Nähe zu denjenigen der generisch ausgerichteten empirischen Unterrichtsforschung aufwiesen. Entsprechend stellte es für das Erstellen dieses Heftes eine große Herausforderung dar, die unterschiedlichen Sichtweisen aufeinander zu beziehen, eine 
gemeinsame Sprache zu etablieren sowie ein vergleichbares, kohärentes Vorgehen zu entwickeln, ohne dabei die Spezifik der beteiligten Disziplinen zu negieren.

Um dieser Herausforderung zu begegnen, wurde zunächst die Bedeutung von Begrifflichkeiten (z. B. naturwissenschaftliche Denk- und Arbeitsweisen, Pädagogische Perspektiven des Sports, historisches Denken), die in der eigenen Disziplin als anerkannt gelten, für die anderen Disziplinen expliziert und ein zyklischer Prozess durchlaufen, um ein geteiltes Begriffsverständnis anzubahnen. Die Idee, eine gemeinsame Struktur über alle Beiträge hinweg zu wählen, mit der der jeweilige Forschungsansatz und -stand verglichen werden kann, stellte sich in der interdisziplinären Arbeit als sehr hilfreich dar. Allerdings wurde auch deutlich, dass einige Aspekte nur begrenzt kompatibel zwischen den unterschiedlichen Ansätzen waren. So handelt es sich beispielsweise beim naturwissenschaftlichen Experiment aus Sicht der anderen Fächer und der allgemeinen Unterrichtsforschung um eine Methode, während es aus Sicht der Naturwissenschaftsdidaktiken auch als Qualitätsmerkmal angesehen wird. Entsprechend waren an einigen Stellen in der gemeinsamen Arbeit Kompromisse notwendig; zu einzelnen Aspekten konnte zudem keine für alle Seiten zufriedenstellende Lösung gefunden werden. Ein Beispiel hierfür stellt die kognitive Aktivierung dar, deren zentraler Bestandteil im Verständnis der allgemeinen Unterrichtsforschung die Anregung tiefenverarbeitender Denkprozesse auf Seiten der Schülerinnen und Schüler darstellt. Im Fach Sport hingegen erscheint durch die enge Verknüpfung kognitiver und motorischer Abläufe eine gemeinsame Behandlung beider Aspekte als kognitiv-motorische Aktivierung sinnvoll.

Bei den Vorbereitungen für die fachspezifischen Reviews stellte sich zudem heraus, dass der jeweilige Forschungsstand in den einzelnen Fächern unterschiedlich umfänglich ausfällt, was auch an der gemeinsamen Betrachtung der naturwissenschaftlichen Fächer lag: Im Literaturreview wurden zwischen 23 (Geschichte) und 95 (Naturwissenschaften) relevante Publikationen identifiziert. Zudem unterschied sich das Verhältnis theoretisch-konzeptueller zu empirischen Arbeiten zwischen den Fächern und umfasste in Geschichte deutlich mehr theoretisch-konzeptuelle Arbeiten als dies in den Naturwissenschaften der Fall war. Auch die Ansätze und Theorien, auf die in den Publikationen Bezug genommen wird, variierten deutlich. Ein einheitliches Vorgehen bei der Erarbeitung der Forschungsstände im Rahmen der fachspezifischen Reviews wurde daher von den Fachvertretern als nicht zielführend betrachtet und es wurden entsprechend unterschiedliche Vorgehensweisen gewählt. Die Erfahrungen weiterführender Diskussionen sowie Anregungen aus einem GEBF-Symposium 2019 zum Thema wurden dann in einem weiteren zweitägigen Workshop 2019 in Potsdam vertieft und weitergeführt sowie abschließende Entscheidungen für den vorliegenden Thementeil in der Zeitschrift Unterrichtswissenschaft getroffen.

Zusammenfassend stellt die Arbeit der Arbeitsgruppe ein gutes Beispiel für die Heterogenität in den Begrifflichkeiten, Strukturierungen und Ansätzen bei der Erforschung von Unterricht in der empirischen Unterrichtsforschung dar. Sie bietet erste Ansätze zu einer stärkeren Vereinheitlichung, zeigt gleichzeitig aber auch die Herausforderungen auf, die mit Versuchen der Abstrahierung von eigenen Ansätzen und Begrifflichkeiten einhergehen sowie mit den entsprechenden Bemühungen um Kompromisse. Mit den Publikationen in diesem Thementeil wurde durch die 
Dokumentation von Herausforderungen und Ergebnissen der gemeinsamen Arbeit ein erster Schritt in der interdisziplinären Arbeit erreicht, der als Grundlage für weiterführende Forschung dienen kann.

\section{Zielstellungen und Struktur des Thementeils}

Konkret ist der vorliegende Thementeil mit seinen insgesamt fünf Beiträgen primär auf die Bearbeitung von drei Zielstellungen ausgerichtet:

1. eine Aufarbeitung des jeweiligen aktuellen Forschungsstandes zu Unterrichtsqualität in den ausgewählten Fächern der Naturwissenschaften, des Sports und der Geschichte;

2. eine Verknüpfung des aktuellen Diskurses in der generisch ausgerichteten empirischen Unterrichtsforschung mit fachdidaktischen Diskursen über den Mathematikunterricht hinaus;

3. eine Auseinandersetzung mit den Gemeinsamkeiten und Spezifika von Aspekten der Unterrichtsqualität in unterschiedlichen Fächern.

Um einen gemeinsamen Bezugspunkt zwischen den Fächern zu ermöglichen, wird in Beitrag 1 von Praetorius, Rogh und Kleickmann zunächst die Notwendigkeit einer Erweiterung des im deutschsprachigen Raum in der generisch ausgerichteten Unterrichtsforschung populären Modells der drei Basisdimensionen von Unterrichtsqualität thematisiert und ein aktuelles Syntheseframework zentraler Aspekte der Unterrichtsqualität mit Bezug auf den Mathematikunterricht vorgestellt. Anschließend wird für jedes der drei Fächer/Fächergruppen ein Review des fachspezifischen Forschungsstandes zu Unterrichtsqualität vorgenommen sowie über das oben genannte Syntheseframework ein Bezug zum aktuellen Diskurs der generisch ausgerichteten empirischen Unterrichtsforschung hergestellt (Beiträge 2-4 von Heinitz \& Nehring; Herrmann \& Gerlach; Zülsdorf-Kersting). Beitrag 5 von Praetorius, Herrmann, Gerlach, Zülsdorf-Kersting, Heinitz und Nehring stellt eine Synthese über die drei fachspezifischen Beiträge dar und schließt mit einem Ausblick auf zukünftige Forschung zu Unterrichtsqualität.

Die Beiträge beruhen auf der gemeinsamen Arbeit der oben beschriebenen Gruppe und sind damit inhaltlich stark vernetzt. Um den hier angestoßenen Diskurs umfänglich wahrnehmen zu können, empfehlen wir, die Lektüre entsprechend der Nummerierung vorzunehmen. Beitrag 1 zur Notwendigkeit der Erweiterung der Basisdimensionen ermöglicht das Verständnis des in allen Beiträgen genutzten Syntheseframeworks. Die Fachbeiträge 2-4 konkretisieren die spezifischen Zugänge der fachdidaktischen Disziplinen und zeigen deren Ergebnisse detailliert auf. Erst dann erschließt sich der abschließende Synthesebeitrag 5 in vollem Umfang.

Danksagung Wir bedanken uns bei Knut Neumann für die Unterstützung bei der Organisation des Begutachtungsprozesses und die hilfreichen Anmerkungen zu den Manuskripten. 


\section{Literatur}

Blömeke, S., \& Olsen, R. V. (2019). Consistency of results regarding teacher effects across subjects, school levels, outcomes and countries. Teaching and Teacher Education, 77, 170-182.

Brunner, E. (2018). Qualität von Mathematikunterricht: Eine Frage der Perspektive. Journal für Mathematik-Didaktik, 39(2), 257-284.

Helmke, A. (2015). Unterrichtsqualität und Lehrerprofessionalität. Diagnose, Evaluation und Verbesserung des Unterrichts. Seelze: Klett.

Klieme, E. (2019). Unterrichtsqualität. In M. Harring, C. Rohlfs \& M. Gläser-Zikuda (Hrsg.), Handbuch Schulpädagogik (S. 393-408). Münster: Waxmann.

Kunter, M., \& Ewald, S. (2016). Bedingungen und Effekte von Unterricht: Aktuelle Forschungsperspektiven aus der pädagogischen Psychologie. In N. McElvany, W. Bos, H. G. Holtappels, M. M. Gebauer \& F. Schwabe (Hrsg.), Bedingungen und Effekte guten Unterrichts (S. 9-31). Münster: Waxmann.

Maurer, C. (2018). Qualitätsvoller Chemie - und Physikunterricht - normative und empirische Dimensionen. In C. Maurer (Hrsg.), Gesellschaft für Didaktik der Chemie und Physik Jahrestagung in Regensburg 2017. Regensburg: Universität Regensburg.

Praetorius, A.-K., \& Charalambous, Y.C. (2018). Classroom observation frameworks for studying instructional quality: looking back and looking forward. ZDM Mathematics Education, 50(3), 535-553.

Praetorius, A.-K., Vieluf, S., Saß, S., Bernholt, A., \& Klieme, E. (2016). The same in German as in English? Investigating the subject-specificity of teaching quality. Zeitschrift für Erziehungswissenschaft, 19, 191-209. 